No recurrence has taken place since the excision of the eye ten years ago and the child is reported perfectly well.

Family History. In no case was there any evidence of a familial or hereditary tendency to glioma retinae.

I wish to thank the members of the surgical staff at Moorfields for the permission to make use of their case records and the facilities given me as Research Scholar to collect the details shown in this paper.

\title{
THE AFTER RESULTS OF CORNEO-SCLERAL TREPHINING FOR GLAUCOMA
}

BY

\author{
R. C. DAVENPORT \\ MOORFIELDS RESEARCH SCHOLAR
}

THE following paper is made up entirely on examination of the results of the corneo-scleral trephine operations performed at Moorfields by the present surgical staff during the five years 1919-1923.

All those cases where the records are available have been included and probably the numbers represent almost all such operations done.

The review is presented quite candidly with no bias for or against the operation. The records kept were nade in the usual rush of the hospital's work, and not with a view to criticai examination later. Accordingly, in many cases they lack of necessity the completeness that a critical examination might desire. Many of them cease abruptly so that the result can only be judged at a point shortly after operation. Others are carried on for some years from operation and thus are of greater value. It is true that the latter are mostly those in which vision was grossly impaired at operation-a fact which has presumably made the patient more zealous in attendance so that further decline might be guarded against if possible. The early glaucoma case with good vision ceases attendance, in many cases, soon after a successful operation, and it seems justifiable to suppose the success of the operation to be prolonged, if not actually total, where the patient does not return to complain of any departure from the satisfactory condition noted soon after operation.

In judging the effects of the operations it has been necessary to rely, as said before, on scanty records. These embrace chiefly the tension of the eye to fingers and the visual acuity. Perimetric records have been far too limited to be used in judgment; indeed where the vision has remained as before the operation and essentially full, and the tension normal, the fields have not, as a rule, been charted, and the few that are shown do not suggest 
that coincident with other satisfactory features, there is a gross loss of visual field.

In all 536 records were investigated. Nearly 100 of these were trephine operations in cases not obviously primary glaucoma. These are made up of secondary glaucoma from irido-cyclitis, dislocated lens, vitreous forwards after lens extraction, old interstitial keratitis, some cases of buphthalmos, etc. All these are excluded in the more critical part of the review which is meant to show the actual results in primary glaucoma. At the same time it seems certain that in many cases of increased tension obviously not the result of primary glaucoma, a trephine operation has been very successful in lowering and keeping lowered for a considerable time at least, the raised tension. Another feature which comes out is that although the eye trephined may have been liable to relapses of irido-cyclitis over many years it seems to stand a trephine operation well and not to react by an immediate sharp exacerbation of the inflammation. In view of the frequency with which a trephine operation in an eye never previously the subject of iritis is followed by quiet inflammation this seems strange. Also it is a little surprising perhaps how often the trephine hole escapes blockage in an eye so constantly throwing out exudate, in view of the fear very often felt that the hole will become blocked by uvea or exudate in a perfectly quiet eye.

The table below shows in broad outline the condition of 405 eyes trephined for primary glaucoma-a few in acute and subacute cases, most in the chronic types. This number includes all the cases where the record is of any value and where the condition of raised tension was not obviously secondary. The cases have been divided into three groups under the visual acuity of the eye, viz. : Group A 6/6-6/12; Growp B 6/18-6/60; Group C less than 6/60.

$\begin{array}{rccc}\text { Before operation. } & \begin{array}{c}\text { In Group A } \\ \text { when last } \\ \text { recorded. }\end{array} & \begin{array}{c}\text { In Group B } \\ \text { when last } \\ \text { recorded. }\end{array} & \begin{array}{c}\text { In Group C } \\ \text { when last } \\ \text { recorded. }\end{array} \\ \text { Group A. } 154 & 124(80.5 \%) & 23(15 \%) & 7(4.5 \%) \\ \text { Group B. } 104 & 31 & 57 & 16 \\ \text { Group C. } 147 & \underline{20} & \underline{26} & \underline{101} \\ \text { Total } \overline{405} & \underline{175} & \underline{106} & 124\end{array}$

The figures need a little examination and review.

Firstly, the 20 cases that improved after operation from less than $6 / 60$ to better than $6 / 12$ were mostly, probably all, cases of acute or subacute glaucoma where the poor visual acuity was due to circulatory interference and haze in the media rather than to damage to fibres of the optic nerve from pressure. If these cases be excluded it will be seen that the results show :
A. 154 before operation
B. 104 ,
155 after operation
C. 127 ,
,
106 ,
,
124 ,
," 
There are the individual cases altering somewhat in acuity, but the figures above are somewhat striking in showing how, in the main, the visual acuity is not greatly altered for better or worse by a trephine operation in chronic glaucoma. That it should be greatly improved is not to be expected where the nerve fibres are damaged, but that the general result shows that it is not lowered by the operation is sound evidence in support of its efficacy. It would seem to show that the mutilation required for maintaining permanent drainage, and thus reasonably normal tension, is minimal. Also it must show how in the great majority of cases the object-viz., the maintenance of such tension-is achieved, for otherwise greater decline of vision would be shown.

Group A comprises of course the cases favourable for operation where the visual acuity is still nearly normal-symptoms are present, tension often raised, and some alterations always present in the visual fields-either peripheral or paracentral.

Of these cases 80 per cent. were apparently successes and only 4.5 per cent. complete failures.

These figures seem very good in view of the possible failures both from progress of the condition and from such events as operation accidents, infection, etc. The patients are mostly in later middle age or actually aged, and are not from the general point of view entirely suitable subjects, and yet such good figures are obtained. The seven failures included three of infection, the others being eyes that steadily went down in spite of repeated trephining, and other operations for relieving the raised tension.

The operation in use is the typical Elliot operation performed with the $1.5 \mathrm{~mm}$. trephine. Two slight departures have been used to some extent, viz., complete iridectomy instead of the peripheral buttonhole and finally stitching the flap carefully back along its whole length to make an early watertight union with a view to securing rapid widespread filtration and oedema beneath the conjunctiva. A review of the untoward effects of the operation is covered by the following table.

\section{Immediate.}

Vitreous loss.

Loss of the disc of sclera into the eye.

Haemorrhage (into vitreous or in fundus).

Prolapse of iris.

Detachment of retina and choroid.

Infection (quiet iritis, acute infection).

Mania.

Remote.

"Late" infection.

Poor drainage and increased tension.

Cataract. 
Vitreous Loss.-There are seven cases in which this is reported. All were cases of very advanced glaucoma and nearly all senile patients. Some of the eyes quietened down not getting any sufficient increasing tension to require excision of the eye. Three of them could only be relieved by excision of the eye. In no case of a middle aged patient with early glaucoma and good vision was vitreous lost.

Loss of disc in the eye.-This is only noted in three cases though probably more common and there is no evidence that it produced any ill effects whatever.

Haemorrhage.-One elderly woman had an extensive haemorrhage into the vitreous after operation. Eight patients-almost all elderly-viz., over 60 -are noted to have shown haemorrhages in the fundus after operation. Some of these cleared quickly-in others there were further fundus changes around the macula with associated decline in visual acuity. This number seems reasonably small in view of their ages and the liability of all such patients to get haemorrhages and fundus changes from vascular disease and senile lesions quite apart from any operation.

Iris prolapse.-In two cases this is noted where no iridectomy was done at operation. In one case it immediately followed the operation-the prolapse was excised and the eye was left with normal tension and $6 / 18$ vision.

In the other case it followed long after operation and there was infection, the case coming under the "late" infections recorded below.

Detachment of choroid.-This is only recorded in ten cases, and in all, the detachment rapidly subsided and later examination showed a normal fundus picture from this aspect. Its effect on vision appeared almost nil, the end result showing the acuity roughly the same as before operation.

\section{Infection}

(1) Quiet iritis.-This appears to take place in the great majority of cases. In spite of the immediate use of atropin after operation, most cases show a little iritis. In a few cases where perhaps no atropin has been used the pupil has become extensively bound by posterior synechiae, but in the great majority the iritis has been shown only by a little uveal deposit on the lens with sometimes very slight adhesion between capsule and iris. The effects of the mild iritis seem very slight-this examination of the results of the operation was partially started with a view to seeing how often the 6/6 eye, though perhaps put into a state of safety by drainage, 
was left with vision lowered to say $6 / 12$ by the effects of the iritis. So little exudate must be produced that the acuity is very seldom measurably lowered. Group A admittedly lumps together all cases with V.A. $6 / 12$ or better, but examination of the details shows little evidence that there is a small but perceptible decline in acuity in full vision eyes as a result of the quiet inflammation which almost always appears to take place. Nor does there appear sufficient exudate thrown out to cause blockage of the small peripheral iridectomy or the trephine hole. There appears little or no evidence that the cases where drainage is impaired have had more serious production of exudate from the iris than the cases with sound drainage.

(2) Acute Infection.-Two cases of acute infection immediately after operation are recorded and in one the eye was lost, the other quietening down.

Mania.-Two or three very elderly patients are reported to have become mentally deranged while in hospital, but as the restriction is much less than after cataract extraction so the liability to mania is accordingly less.

\section{Remote}

Late infections.--In all, fourteen cases of "late" infection were treated during the years under review. Some of these were operated on in years previous to 1919 -others during the five years recorded. To quote all these against the number of trephine operations done in this time seems a fair way of measuring the frequency of this misfortune. The majority of cases occurred a few months after the operation-one case as late as seven years after. The type of infection varied-in some there was a leaking or staining area over the trephine hole with acute iritis, hypopyon or even panophthalmitis, in others there was a quieter type of plastic inflammation not so easily traced to infection from the trephine hole and in one case definitely associated with a generalized toxaemia producing widespread rheumatoid arthritis. Thus it seems that some at least are accidental in so far as an irido-cyclitis happens to be set up in an eye previously trephined, the infection being due to a blood toxaemia and not to a liabilitv to infection through a hole connecting the inside of the globe with the outside. In eight of the cases however it is clearly an infection via the trephine hole.

Serious and unfortunate as the accident is, the result is by no means hopeless. Most of the cases occurred in old people, eleven out of fourteen being over 60. years, and yet of them all, evisceration was only necessary in two cases and when on treatment 
the inflammation had subsided six of them were left with vision $6 / 12 \mathrm{pt}$. to $6 / 18 \mathrm{pt}$. In two or three of the others lens opacity was the cause of impaired vision after the inflammation and this may have been quite independent of the infection. Thus the late infection, while a serious if not very frequent misfortune, is hardly the bogey it is often made out to be with regard to the total abandonment of the trephine operation.

One of the cases is of interest in so far as infection came about in a 6/18 eye some six months after trephining. On treatment of the kerato-irido-cyclitis it all settled and he was left with vision little impaired, viz., 6/18 pt. Two years later his other eye also $6 / 18$ from glaucoma, was operated on by Holth's "punch operation." Two months later this eye became acutely inflamed and though the inflammation subsided it relapsed later so severely that excision was performed.

Poor drainage and relapse of tension.-In the great majority of cases one trephine operation has left the eye with normal tension up to the last record available. In a few cases, however, in spite of an apparently successful operation, drainage was never satisfactory and increased tension continued. In some of these a second operation was at once successful, in others many operations-three or four trephinings sclerotomy, iridectomy-all failed to produce normal tension and the vision failed steadily. There seems to have been no constant feature of the cases with regard to age, history or appearance of the condition. At the same time it is quite obvious in looking through the records that the older patients do not do so well. In some there is clearly maintained high tension-in others it is only that vision declines slowly in spite of an apparently safe tension. In some of these there is an obvious cause such as lens sclerosis or fundus changes - vascular or degenerative. But in several cases of the senile glaucoma type-patients over 65 years say-there has been steady loss of vision with shrinking fields and increased excavation and pallor of the discs in the presence of a normal tension.

The records would suggest a "malignant" type at any age which is hard to check and control, and in addition a type that may come under such a heading as "senile cavernous atrophy of the discs," where the tension does not appear raised and where drainage does not check the progress of the condition as in a more straightforward case. Only a few can be put in such a class and all are old people. The available records, however, do not make it possible to differentiate them at all sharply.

Cataract.-There is little or no evidence that the trephine operation predisposes to cataract. No eye of class A developed cataract as a sequel to the operation. Some of the older people were found 
to have lens opacity at operation, others after operation, but there is no evidence that the operation can be held responsible. In some, as would be expected, lens opacity appeared some years after a successful trephine operation in an elderly patient. In some of the cases that did not do well cataract developed, suggesting that an imperfect or unsuccessful operation was much more likely to be followed by cataract than a successful one.

The results of extraction of the lens after a trephine operation do not seem successful as far as vision goes. There are only three or four isolated cases and in all these vision was poor after the extraction, successful though the operation was. At the same time vision was not good before the cataract developed-as far as the records can show.

This paper is the outcome of part of my work as Moorfields Research Scholar, and I have had free use of all the patients' records.

For such facilities and for permission to publish the results I am indebted to the surgical staff whom I wish tc thank.

\title{
ON THE VISIBILITY OF THE NUCLEI OF THE ENDOTHELIAL CELLS OF THE CORNEA IN MAN
}

BY

\author{
T. HARRISON BUtLER \\ BIRMINGHAM
}

THE nuclei of the endothelial cells are easily seen in the eye of the pig, but it is generally held that they are invisible in the human cornea.

I have just examined an engineer, aged 51 years, who in his younger days had been a miner. His endothelial cells were unusually large and I was astonished to find that the nuclei were perfectly visible. Using ocular 2 and objective A3, a magnification of 35 , the cells were seen to be hexagonal and in the centre of each there was a dark dot. This did not alter in appearance with any modification of the focus and was not therefore an optical effect. The appearance was exactly similar to that seen in the pig's eye and I have no doubt that I was looking at the nuclei. I immediately with the same optical system examined another normal patient and was quite unable to see the nuclei and also confirmed the observation that the miner's cells were abnormally large. The nuclei were visible in both corneae. 